\title{
Neglected tropical diseases: survey and geometry of randomised evidence
}

\author{
(C) (1) $\Theta$ OPEN ACCESS
}

\author{
Shanthi Kappagoda fellow ${ }^{1}$, John P A loannidis professor ${ }^{2}$ \\ ${ }^{1}$ Center for Primary Care and Outcomes Research and Division of Infectious Diseases and Geographic Medicine, Stanford University School of \\ Medicine, Stanford, CA, USA; ${ }^{2}$ Stanford Prevention Research Center, Department of Medicine and Department of Health Research and Policy, \\ Stanford University School of Medicine, and Department of Statistics, Stanford University School of Humanities and Sciences, Stanford
}

\begin{abstract}
Objective To assess the quantity and distribution of evidence from randomised controlled trials for the treatment of the major neglected tropical diseases and to identify gaps in the evidence with network analysis.
\end{abstract}

Design Systematic review and network analysis.

Data sources Cochrane Central Register of Controlled Trials and PubMed from inception to 31 August 2011.

Study selection Randomised controlled trials that examined treatment of 16 neglected tropical diseases or complications thereof published in English, French, Spanish, Portuguese, German, or Dutch.

Results We identified 971 eligible randomised trials. Leishmaniasis (184 trials, 23039 participants) and geohelminth infections; 160 trials, 46887 participants) were the most studied, while dracunculiasis (nine trials, 798 participants) and Buruli ulcer (five trials, 337 participants) were least studied. Relative to its global burden of disease, lymphatic filariasis had the fewest trials and participants. Only $11 \%$ of trials were industry funded. Either a single trial or trials with fewer than 100 participants comprised the randomised evidence for first or second line treatments for Buruli ulcer, human African trypanosomiasis, American trypanosomiasis, cysticercosis, rabies, echinococcosis, New World cutaneous leishmaniasis, and each of the foodborne trematode infections. Among the 10 disease categories with more than 40 trials, five lacked sufficient head to head comparisons between first or second line treatments.

Conclusions There is considerable variation in the amount of evidence from randomised controlled trials for each of the 16 major neglected tropical diseases. Even in diseases with substantial evidence, such as leishmaniasis and geohelminth infections, some recommended treatments have limited supporting data and lack head to head comparisons.

\section{Introduction}

The neglected tropical diseases comprise parasitic, viral, and bacterial infections that cause substantial morbidity among the world's poorest people but have historically not been targeted for intensive drug development because of limited financial incentives in the private sector. ${ }^{12}$ These diseases are both a cause and an effect of poverty. Some cause chronic disability (such as lymphatic filariasis and leprosy) and blindness (such as trachoma and onchocerciasis), which impair adults' ability to work, while others (such as geohelminth infections) are associated with decreased educational achievements in children. ${ }^{3}$ More than one billion people worldwide have one or more neglected tropical disease, causing an estimated 534000 deaths and a loss of 57 million disability adjusted life years (DALYs) annually. ${ }^{4}$ Estimates of their global burden of disease might undervalue the importance of neglected tropical diseases to public health, particularly in areas where they co-occur with HIV and malaria. For instance, schistosomiasis infection might enhance HIV transmission ${ }^{5}$ and both schistosomiasis and geohelminth infections might be associated with more rapid progression of HIV disease..$^{5-7}$ Geohelminth infections and schistosomiasis are also associated with increased susceptibility to and severity of malaria. ${ }^{8-11}$

Although some neglected tropical diseases have affordable and effective treatments, many require treatments that are expensive, toxic, and difficult to administer. In addition, some treatment regimens - unchanged for decades — might be declining in efficacy because of drug resistance. ${ }^{12-14}$ Historically, drug development for these diseases has been limited. A review of drug development in the United States and Europe found that of 1393 new drugs developed and marketed between 1975 and 1999 , only $1.1 \%$ were for tropical diseases.$^{15}$ In the past several years, new public-private partnerships, such as the Drugs for 
Neglected Diseases Initiative, have provided a framework to accelerate drug development. ${ }^{16}$

We compiled the evidence from randomised controlled trials for treatment of 16 neglected tropical diseases. We used treatment networks to illustrate the types and numbers of treatments compared (so called "geometry" of evidence). ${ }^{17} 18$ These networks provide a visual and quantitative synopsis of the treatments studied and the amount of randomised evidence supporting current treatment guidelines and indicate whether some treatments or comparisons have been studied disproportionately.

\section{Methods}

\section{Search strategy and eligibility criteria}

We searched PubMed and Cochrane Central Register of Controlled Trials for randomised controlled trials published up to 31 August 2011 that examined treatment of 16 neglected tropical diseases: American trypanosomiasis (Chagas disease), Buruli ulcer, cysticercosis, dengue, dracunculiasis (Guinea worm), echinococcosis (hydatid cyst disease), foodborne trematode infections, geohelminth infections, human African trypanosomiasis, leishmaniasis, leprosy, lymphatic filariasis, onchocerciasis, rabies (prophylaxis after exposure), schistosomiasis, and trachoma. We also reviewed our own literature collections, relevant Cochrane reviews, and references of identified eligible trials to identify additional trials. Appendix table 1 shows the search strategy in detail.

We excluded trials with non-human participants, non-randomised and pseudo randomised trials, and non-treatment trials (such as trials of diagnostic tests, prevention interventions, and pharmacokinetic studies in healthy volunteers). We excluded abstracts, descriptions of planned studies, and subgroup or secondary analyses of previously published randomised controlled trials. We also excluded trials published in languages other than English, French, Spanish, Portuguese, German, or Dutch. Trials were eligible regardless of phase. For preliminary reports of clinical trial data that were later included in a more complete publication, we included only the final publication. For duplicate publications of the same trial we included the publication that appeared first; if both appeared in the same year, we included the publication from the journal with the higher impact factor. Studies that examined more than one of the 16 diseases (such as combination treatment for schistosomiasis and geohelminth infections) were included as a separate randomised controlled trial for each disease.

\section{Data extraction}

For each trial we read the first page, the methods, and the acknowledgments (to identify funding source) and extracted information on first author, publication year, journal, country/site(s), whether the study examined the primary disease or a complication, study design (crossover, cluster, or neither), interventions, number of participants per arm, and follow-up period. The trials were classified as either primary disease trials in which the tested treatments were aimed at eliminating or neutralising (as in rabies post-exposure prophylaxis) the aetiologic agent of the infection, regardless of whether the infection was symptomatic, or complication trials in which patients with a specific complication of the primary infection (for example, anaemia, nutritional deficiency, growth impairment, portal hypertension, or cardiomyopathy) were enrolled. The treatments tested in complication trials generally did not target the infectious agent but aimed to modify the pathophysiology of the complication-for example, use of angiotensin converting enzyme (ACE) inhibitors for cardiomyopathy associated with Chagas disease. We recorded whether the trial had a funding statement and all funding sources (industry, government, or other public agency (including the World Health Organization), charity or foundation, and hospital or university).

\section{Descriptive analyses and correlation with disease burden}

We present descriptive statistics for eligible trials and show the cumulative sample size and number of trials over time. To provide an estimate of how the evidence from randomised controlled trials has changed over time for different diseases, we divided the trials into those published before $2000(n=609)$ and those published in 2000-12 ( $\mathrm{n}=362)$. We chose 2000 as a watershed date as several initiatives to promote drug development for the neglected tropical diseases were started after $2000 .^{16}$

For cluster randomised controlled trials, we used the number of clusters in the sample size calculations. For each disease, we separated trials targeting the primary infection from trials examining disease complications. We examined the correlations between the global burden of disease and the number of trials and total sample size for each disease. We obtained data on the burden of disease in DALYs for American trypanosomiasis, ${ }^{19}$ dengue, ${ }^{19}$ echinococcosis,${ }^{20}$ foodborne trematode infections,${ }^{21}$ human African trypanosomiasis, ${ }^{19}$ leishmaniasis, ${ }^{19}$ leprosy, ${ }^{19}$ lymphatic filariasis, ${ }^{19}$ onchocerciasis, ${ }^{19}$ rabies, ${ }^{22}$ geohelminth infections, ${ }^{19}$ schistosomiasis, ${ }^{19}$ and trachoma. ${ }^{19}$ For three diseases (dracunculiasis, Buruli ulcer, and cysticercosis) we did not have reliable estimates of global burden of disease. DALYs are a measure of the years of life lost from ill health, disability, or early death from disease in a population and are calculated as the years of life lost plus the years lived with disability. ${ }^{23}$ They can be used to compare the burden of disease across diseases that have disparate clinical courses-for example, a short lived disease with high mortality such as rabies and a chronically disabling disease with low mortality such as lymphatic filariasis.

\section{Evidence for first and second line treatments}

For each disease, we recorded the number of trials and total sample size for each treatment. We present these data only for currently recommended first or second line treatments. When available (for Buruli ulcer, ${ }^{24}$ dengue, ${ }^{25}$ echinococcosis, ${ }^{26}$ leprosy, ${ }^{27}$ rabies, ${ }^{28}$ and trachoma ${ }^{29}$ ) we used WHO guidelines published in the past six years to identify first and second line treatments. We used published consensus guidelines for cysticercosis. ${ }^{30}$ For the nine remaining diseases, we reviewed the first and second line treatments and the primary references cited in UpToDate and Medical Letter: Drugs for Parasitic Infections. ${ }^{31}$ When these sources differed or for diseases for which influential randomised controlled trials have changed clinical practice pending clinical guideline revisions, we used our own judgment, additional recent randomised controlled trials, and review articles to designate first and second line treatments. This strategy was used for geohelminth infections (ascariasis, ${ }^{32-37}$ trichuriasis, ${ }^{32}{ }^{38-43}$ hookworm, ${ }^{37}{ }^{44-46}$ strongyloidiasis ${ }^{41474}$ ), onchocerciasis ${ }^{49}$ schistosomiasis, ${ }^{50}$ lymphatic filariasis, ${ }^{49} 51$ visceral, [52-56] cutaneous, ${ }^{57-60}$ and mucocutaneous ${ }^{61}$ leishmaniasis, American trypanosomiasis, ${ }^{62-64}$ foodborne trematode infections (clonorchiasis, ${ }^{65} 66$ opisthorchiasis, ${ }^{66}{ }^{67}$, fascioliasis, ${ }^{68-70}$ fasciolopsiasis, ${ }^{71}$ paragonimiasis $\left.{ }^{72-74}\right)$, human African trypanosomiasis, ${ }^{75-77}$ and dracunculiasis. When there was any uncertainty, we erred on the side of including more rather than fewer recommended 
treatments. Appendix table 2 shows the designation of first and second line regimens for each of these nine diseases.

\section{Treatment networks}

The networks include only primary disease trials, omitting disease complication trials. Each node in a network (circle) represents a treatment and the connecting lines represent randomised comparisons. The node size is proportional to the total sample size for each treatment and the thickness of the connecting line is proportional to the number of comparisons. "Self loops" on the same node are comparisons within drugs - for example, comparisons of the same drug at different doses. We analysed in depth networks for diseases with over 40 randomised controlled trials.

We used methods described elsewhere ${ }^{17}$ to calculate network diversity and co-occurrence metrics. Diversity is a measure of the number of treatments in a network and whether the treatments are equally represented. A network with few treatments will have low diversity. A network where one or two treatments are repeatedly tested while others are tested rarely will have lower diversity than one where treatments are more evenly represented. We used the probability of interspecific encounter (PIE) - probability that two treatments sampled at random without replacement from all possible treatments in a network will be different- to estimate diversity. When there are $\mathrm{N}$ different treatments tested in a total of $\mathrm{S}$ clinical trial arms, the maximum value that the probability of interspecific encounter can take is $\mathrm{S}(\mathrm{N}-1) /(\mathrm{N}(\mathrm{S}-1))$ and the minimum is $(\mathrm{N}-1)(2 \mathrm{~S}-\mathrm{N}) /(\mathrm{S}(\mathrm{S}-1))$, thus it cannot be greater than 1 or less than 0 under any circumstances. When there are many trials (for example, over 40, as in our analysis) and a modest number of different treatments, the maximum potential value of the probability of interspecific encounter is close to 1 and values above 0.8 represent high diversity.

We used the co-occurrence score (or C score) to assess whether some pairs of treatments are compared more or less often than would be expected by chance-that is, whether specific comparisons are preferred and/or others are avoided. To generate the $\mathrm{C}$ score, a symmetrical matrix of all the possible treatments is made by listing all the treatments in the dataset as row and column headings and entering a 1 or 0 in the matrix depending on whether a particular pairwise comparison is represented in the data or not. The row and column totals are then kept constant and multiple (5000) permutations are run to generate multiple possible sets of pairwise comparisons that can be made with the dataset. The observed matrix is then compared with the expected or average value of matrix over multiple permutations to test the null hypothesis that there is no co-occurrence. A permutation based $\mathrm{P}$ value of $<0.05$ for the $\mathrm{C}$ score represents significant co-occurrence. The $\mathrm{P}$ value can be non-significant, even if there is substantial co-occurrence, when the number of trials is small; for this reason, we limited analyses to networks with more than 40 trials.

\section{Software}

We used SAS Enterprise Guide 4.3 (SAS Institute, Cary, NC) for descriptive statistics and Ucinet for Windows Software for Social Network Analysis and NetDraw: Graph Visualization Software (Analytic Technologies, Harvard, MA) for network diagrams. For diversity and co-occurrence calculations, we used EcoSim Professional: Null Modeling Software for Ecologists, version 1 (Acquired Intelligence. Kesey-Bear, and Pinyon Publishing, Montrose, CO).

\section{Results}

\section{Search results}

The initial searches of PubMed and Cochrane Central Register of Controlled Trials generated 2857 publications (fig $1 \Downarrow$ ). We retained 927 publications containing 966 eligible randomised controlled trials. Five publications were added from our literature collection or bibliographies of included publications eventually yielding 971 randomised controlled trials in 932 publications.

\section{Trial characteristics}

Of the 971 trials, 854 dealt with one of the 16 primary diseases and 117 with a complication of one of the diseases (table $1 \Downarrow$ ). The most common study sites were Africa (27.6\%) and Southeast Asia (26.9\%). Only $26(2.7 \%)$ of the trials were multicentre. Less than $3 \%$ and $2 \%$ of the trials had cluster or crossover designs, respectively. Most trials were either publicly funded or had no funding statement; only $11 \%$ reported industry funding. The median number of randomised participants per trial was 89 (interquartile range 42-184).

\section{Randomised evidence and correlation with burden of disease}

Figure $2 \Downarrow$ shows the cumulative number of randomised participants and trials over time. There is an inflection point in the pace of evidence accumulation around 1980 when the growth of evidence accelerates, but growth has remained steady since then. Table $2 \Downarrow$ shows that for six diseases-Buruli ulcer, cysticercosis, dengue, echinococcosis, human African trypanosomiasis, and leishmaniasis - there were more randomised controlled trials published in the past 12 years than in the preceding 50. For the 10 remaining diseases there have been fewer trials published in the past 12 years than in the preceding 50. The correlation coefficient between the two series was 0.66 , indicating there was a modest correlation between the number of trials published before and after 2000 for each of the diseases. Table $3 \Downarrow$ shows the number of trials, total sample size, and annual global burden for each disease. Leishmaniasis was the most studied disease (184 trials, total sample size 23 039), followed by geohelminth infections (160 trials, total sample size 46887 ), and schistosomiasis (142 trials, total sample size 35 026). The least studied diseases were dracunculiasis (nine trials, total sample size 798) and Buruli ulcer (five trials, total sample size 337). There was no strong correlation (Pearson correlation coefficient $0.33, \mathrm{P}=0.27$ for both analyses) between the global burden of disease and total sample size or number of clinical trials (appendix figure 1) for 13 diseases with available burden of disease estimates. Relative to its global burden of disease (5 777000 DALYs), lymphatic filariasis was the least studied disease (73 trials, total sample size 9996). Leprosy was the best studied (120 trials, total sample size 14 772) relative to its fairly low global burden of disease (198 000 DALYs).

Dengue, American trypanosomiasis, the five major foodborne trematode infections, human African trypanosomiasis, dracunculiasis, mucocutaneous leishmaniasis, and Buruli ulcer each had 10 or fewer randomised controlled trials. Few treatment regimens were tested: six for dengue, five for American trypanosomiasis, seven or fewer for each of the foodborne trematode infections, six for dracunculiasis, seven for human African trypanosomiasis and mucocutaneous leishmaniasis, and eight for Buruli ulcer. Diseases with 11-40 randomised controlled trials examining the primary disease included rabies $(n=13)$, strongyloidiasis $(n=14)$, echinococcosis $(n=15)$, 
tuberculoid/paucibacillary leprosy $(n=21)$, trachoma $(n=29)$, and cysticercosis $(n=32)$. As table $4 \Downarrow$ shows the evidence for one of the first or second line treatments for Buruli ulcer (all recommended treatments), human African trypanosomiasis (pentamidine), chronic American trypanosomiasis (nifurtimox), cysticercosis (praziquantel plus corticosteroids), rabies (several intramuscular post-exposure vaccine regimens), echinococcosis (percutaneous drainage plus albendazole and watchful waiting/no treatment), New World cutaneous leishmaniasis (pentamidine), and foodborne trematode infections (nitazoxanide for fascioliasis and praziquantel for clonorchiasis,

paragonimiasis, and fasciolopsiasis) comes from either a single trial or a total of less than 100 randomised participants. Dengue and dracunculiasis have been investigated in few and primarily small randomised controlled trials, but do not have currently recommended pharmacological treatments. Appendix table 3 shows the amount of evidence for each treatment for each disease and disease complication.

\section{Treatment networks}

Figure $3 \Downarrow$ shows network diagrams of the tested regimens and comparisons for the 10 disease entities with more than 40 randomised controlled trials-schistosomiasis, ascariasis, hookworm, trichuriasis, lymphatic filariasis, visceral leishmaniasis, Old World cutaneous leishmaniasis, New World cutaneous leishmaniasis, onchocerciasis, and multibacillary leprosy. Appendix figure 2 shows networks for diseases that had 11-40 randomised controlled trials. Figure 3 omits a few trials that used regimens that were not compared with any of those shown in the connected network. This included one trial for Old World cutaneous leishmaniasis, three for visceral leishmaniasis, one for ascariasis, one for schistosomiasis, four for multibacillary leprosy, and one for lymphatic filariasis. Data from these trials are included in tables 3 and 4, but were not used to calculate the probability of interspecific encounter and C scores.

The typical geometry resembled a star or several stars, where star centres were usually first line treatments, sometimes second line treatments, and often placebo. There were some cases where treatments that are not accepted first or second line treatments were star centres, meaning a drug that is not a current first or second line drug has been compared against multiple other treatments. In most cases, this just reflects changes in clinical practice over time. These star centres are either treatments that were recommended treatments at the time of the trial—such as DEC (diethylcarbamazine) for onchocerciasis, ${ }^{78} 79$ bephenium for hookworm,${ }^{80}$ and dapsone monotherapy for leprosy ${ }^{81}$ - or a standard of care in the country where the trial was conducted at the time of the trial-such as dapsone-rifampin dual treatment for multibacillary leprosy in Asia. ${ }^{82} 83$ Other drugs such as ivermectin and albendazole monotherapy for lymphatic filariasis were shown to be effective in small randomised controlled trials and were used as comparators (often with combination treatment) in subsequent larger trials. ${ }^{84} 85$

For nine of the 10 disease networks in figure 3, there was more than one first or second line treatment. In seven of these nine, one or two treatments were most often studied while the other recommended treatments had limited evidence. For example, recommended treatments were compared in fewer than seven clinical trial arms and/or fewer than 1000 patients in onchocerciasis (combination ivermectin-doxycycline), trichuriasis (ivermectin), New World cutaneous leishmaniasis (pentamidine and miltefosine), and visceral leishmaniasis (miltefosine, paromomycin, amphotericin B lipid complex, and liposomal amphotericin).
In many networks, there were few data from head to head comparisons for first and second line treatments, despite them being tested in many trials against multiple comparators. The most extreme examples of this are in the networks for New World cutaneous leishmaniasis, for which there were no head to head comparisons between second line treatments miltefosine and pentamidine, and in visceral leishmaniasis, for which there were few or no head to head comparisons between miltefosine and paromomycin and between miltefosine and either amphotericin B lipid complex or liposomal amphotericin. To a lesser extent, this lack of head to head comparisons between recommended treatments is shown in the geohelminth networks. Trichuriasis and ascariasis networks have few or no comparisons of mebendazole and ivermectin, and the hookworm network shows few comparisons of pyrantel pamoate with albendazole or mebendazole.

All 10 networks had high diversity, with probability of interspecific encounter scores $\geq 0.82$ (table $5 \Downarrow$ ). In seven of the 10 networks (Old World leishmaniasis, visceral leishmaniasis, the three geohelminth infections, multibacillary leprosy, and lymphatic filariasis) there was significant co-occurrence, meaning there was a tendency to compare specific pairs of treatments more often and to avoid other comparisons. This could reflect the fact that these networks have multiple star centres and each star centre is compared against a largely non-overlapping set of comparators. Conversely, the networks for New World cutaneous leishmaniasis and schistosomiasis, which mostly have a single star centre (systematic pentavalent antimonials and praziquantel, respectively), do not exhibit significant co-occurrence.

\section{Discussion}

In this systematic review, we identified almost a thousand randomised controlled trials examining the 16 neglected tropical diseases, although the distribution of the evidence across diseases was uneven. Six diseases had $\leq 10$ randomised controlled trials, and for eight diseases either a single trial or fewer than 100 participants comprised the randomised evidence for a first or second line regimen. Even for diseases with numerous randomised controlled trials, such as leishmaniasis and geohelminth infections, evidence was limited for some of the recommended treatments, and there were few head to head comparisons for diseases with more than one recommended treatment. As expected, trials funded by the pharmaceutical industry were uncommon.

Several reasons could explain the lack of randomised evidence for some recommended treatments. For some diseases, a recommended treatment might not be available in all endemic areas-for example, the schistosomiasis drug oxamniquine is mainly used and available in Brazil.$^{86}$ Oxamniquine is effective only against $S$ mansoni, which limits its utility in areas of Africa where more than one species of Schistosoma co-occur. Other recommended treatments are unaffordable relative to standard treatment-for example, miltefosine and lipid formulations of amphotericin for visceral leishmaniasis. Despite cost and despite limited clinical evidence, however, newer treatments might rapidly supplant previously recommended drugs when they have seemingly much better side effect profiles, ease of administration, or drug resistance profiles than standard treatment (for example, miltefosine compared with pentavalent antimonials in visceral leishmaniasis). The relative lack of evidence from randomised controlled trials for certain diseases might also reflect the geographical distribution of the disease and whether there is capacity for clinical trial research in 
endemic sites-for example, through links with institutions in developed countries.

Trials with inactive controls are typically less expensive and require smaller sample sizes than head to head comparisons. This could explain the popularity of placebo controlled trials in resource poor settings. The choice of placebo or "strawman" comparators leading to a lack of head to head comparisons of appropriately dosed recommended treatments has been noted in other diseases ${ }^{87-90}$ and has often been attributed to the goals of industry sponsors. ${ }^{87-91}$ In our data, however, where industry funding was uncommon, lack of head to head comparisons could be explained by the cost and availability constraints noted above as well as unwillingness to use older, more toxic drugs as comparators with newer, seemingly safer agents. This might explain the lack of head to head comparisons between newer drugs and pentamidine in New World leishmaniasis. Finally, placebo controlled trials can sometimes still be appropriate in self limited diseases such as Old World cutaneous leishmaniasis. In general, however, head to head comparisons are essential to understand the relative effectiveness and harms of currently recommended regimens. Such comparisons of active agents are not lacking in general for neglected tropical diseases; it is specifically head to head comparisons of the most promising recommended agents that are sparse. Our networks show that often each recommended treatment forms the centre of a star whose points represent a cadre of several inferior regimens. There is little overlap between the comparators of each recommended treatment, and trials are rarely designed to establish which recommended regimen is best. Lastly, as the field of neglected tropical disease control moves towards integrated programmes of combined preventive chemotherapy, ${ }^{1392}$ it is notable how few published randomised controlled trials examined concurrent treatment of more than one disease with combination chemotherapy_-just 13 trials in our dataset (mostly geohelminth infections and schistosomiasis or geohelminth infections and lymphatic filariasis).

\section{Limitations}

Our work has several limitations. Exclusion of unpublished trials and trials published only in abstract underestimates the amount of randomised evidence. The published evidence, however, already gives us a wide view of the topic. While publication bias can affect estimates of treatment effects, it is unclear whether and how it would affect network geometries. Testing for co-occurrence is subject to power considerations, but our networks had a sufficiently large number of trials to make these estimates robust. The number of nodes in each network has some subjectivity; other investigators might have combined more treatments into single categories, which would have yielded simpler network patterns and lower estimates of network diversity. The correlations between the DALY based global burden of disease estimates and the amount of data from randomised controlled trials should be interpreted with caution. The established disability weights that go into the DALY estimates for the different neglected tropical diseases might not be nuanced enough to capture the effects of the wide range of different acute and chronic disease manifestations caused by the diseases, and inappropriate disability weights can considerably underestimate disease burden..$^{93}$ In addition, for several diseases there is a paucity of literature on which to base accurate estimates of disease specific mortality.

Finally, we did not perform a quantitative synthesis of treatment effects. This would have been impractical for a body of evidence including almost 1000 trials. Instead, we mapped the current perceptions about recommended treatments and the amount of evidence supporting them. Network meta-analyses would benefit from our collected evidence to estimate precise treatment effects for these multiple different treatments.

\section{Conclusions}

Our systematic overview depicts the randomised evidence for the treatment of neglected tropical diseases and highlights where opportunities and needs exist for future trials. Clearly, there is not a direct inverse correlation between the amount of evidence available for each disease and the need for new drug development. Our findings must be interpreted in light of the safety and efficacy of existing treatments and other disease control efforts. For example, dracunculiasis is currently the target of a successful prevention oriented eradication programme ${ }^{94}$; initiation of treatment focused research and development is unlikely to be a good use of resources. This situation contrasts with human African trypanosomiasis and visceral leishmaniasis, for which data on the safety and efficacy of existing drugs are limited, and with the foodborne trematode infections, for which well tolerated drugs are available but the evidence supporting their use and head to head comparisons among recommended treatments is minimal. Box 1 lists disease attributes - in addition to "relative neglect" and burden of disease - that could be used to determine priorities in drug development for neglected tropical diseases. Network analyses can provide a way to see not only which diseases are most and least studied but also which direct comparisons are absent or under-represented in the evidence from randomised controlled trials (box 2). Our systematic presentation of the randomised evidence for 16 neglected tropical diseases might help to prioritise research and advocacy efforts and provide a way to measure whether public-private partnerships and incentive programmes for research and development are succeeding in expanding the treatment options for these diseases.

Contributors: Both authors conceived the study, analysed the data, interpreted the results, drafted the manuscript, and approved the final version. SK extracted the data with help from JPAI. JPAI is guarantor. Funding: SK was supported in part by grant HS000028 from the Agency for Healthcare Research and Quality. The content is solely the responsibility of the authors and does not necessarily represent the official views of the Agency for Healthcare Research and Quality. The funding source had no role in the design or implementation of the study or the decision to publish.

Competing interests: Both authors have completed the ICMJE uniform disclosure form at www.icmje.org/coi_disclosure.pdf (available on request from the corresponding author) and declare: no support from any organisation for the submitted work; no financial relationships with any organisations that might have an interest in the submitted work in the previous three years; no other relationships or activities that could appear to have influenced the submitted work.

Ethical approval: Not required.

Data sharing: Dataset of the primary raw data is available on request from the corresponding author.

Molyneux DH, Hotez PJ, Fenwick A. "Rapid-impact interventions": how a policy of integrated control for Africa's neglected tropical diseases could benefit the poor. PLOS Med 2005;2:e336.

2 Hotez PJ, Molyneux DH, Fenwick A, Kumaresan J, Sachs SE, Sachs JD, et al. Control of neglected tropical diseases. N Engl J Med 2007;357:1018-27.

3 Hotez PJ, Fenwick A, Savioli L, Molyneux DH. Rescuing the bottom billion through control of neglected tropical diseases. Lancet 2009;373:1570-5.

4 Hotez PJ, Molyneux DH, Fenwick A, Ottesen E, Ehrlich Sachs S, Sachs JD. Incorporating a rapid-impact package for neglected tropical diseases with programs for HIV/AIDS, tuberculosis, and malaria. PLoS Med 2006;3:e102.

5 Secor WE. The effects of schistosomiasis on HIV/AIDS infection, progression and transmission. Curr Opin HIV AIDS 2012;7:254-9. 


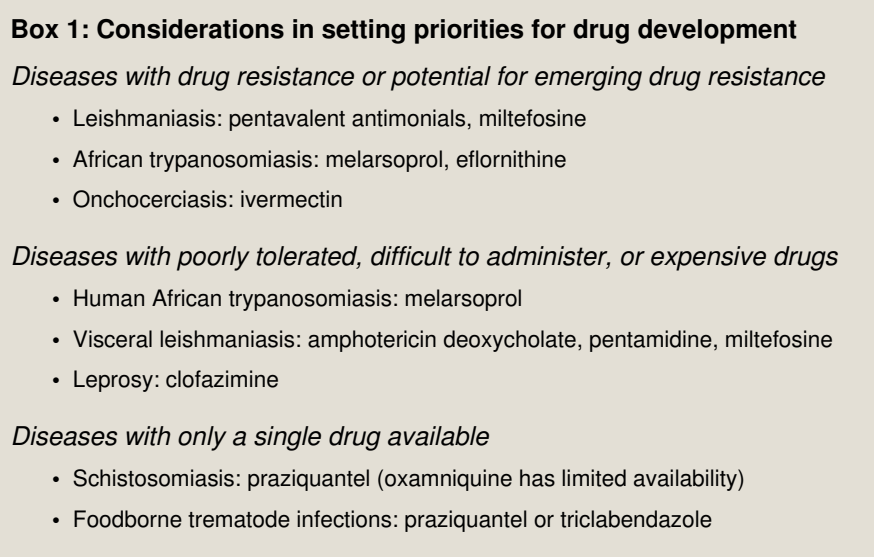

\section{Box 2: Examples of head to head comparisons among first and/or second line treatments that have been conducted} only once or not at all in published randomised trials

Cutaneous leishmaniasis

- Liposomal amphotericin/amphotericin B lipid complex $v$ miltefosine

- Combination of miltefosine with parenteral treatments $v$ amphotericin B lipid complex/liposomal amphotericin alone

- Heat treatment $v$ topical aminoglycosides (Old World cutaneous leishmaniasis only)

Mucocutaneous leishmaniasis

- Miltefosine $v$ amphotericin deoxycholate/liposomal amphotericin/paromomycin

Visceral leishmaniasis

- Miltefosine $v$ liposomal amphotericin/paromomycin

Trichuriasis

- Mebendazole $v$ ivermectin

\section{Echinococcosis}

- Percutaneous drainage $v$ drainage plus albendazole/mebendazole

Buruli ulcer

- Shorter durations of streptomycin plus rifampin $v$ standard duration

\section{What is already known on this topic}

Diseases described as "neglected" have historically not been targeted for research and drug development

These diseases carry a high cumulative global burden of disease

\section{What this study adds}

We identified 971 randomised trials pertinent to the treatment of neglected tropical diseases and found large variability, with some diseases being far more understudied than others

For several neglected diseases evidence for at least one first or second line treatment was scant and head to head comparisons were uncommon

6 Mbabazi PS, Andan O, Fitzgerald DW, Chitsulo L, Engels D, Downs JA. Examining the relationship between urogenital schistosomiasis and HIV infection. PLoS NegI Trop Dis 2011; $5:$ e1396

7 Fincham JE, Markus MB, Adams VJ. Could control of soil-transmitted helminthic infection influence the HIV/AIDS pandemic. Acta Trop 2003;86:315-33.

8 Nacher M, Singhasivanon P, Yimsamran S, Manibunyong W, Thanyavanich N, Wuthisen $\mathrm{R}$, et al. Intestinal helminth infections are associated with increased incidence of Plasmodium falciparum malaria in Thailand. $J$ Parasitol 2002;88:55-8.

9 Spiegel A, Tall A, Raphenon G, Trape JF, Druilhe P. Increased frequency of malaria attacks in subjects co-infected by intestinal worms and Plasmodium falciparum malaria. Trans R Soc Trop Med Hyg 2003;97:198-9.

10 Druilhe $\mathrm{P}$, Tall A, Sokhna C. Worms can worsen malaria: towards a new means to roll back malaria? Trends Parasitol 2005;21:359-62.

11 Sokhna C, Le Hesran JY, Mbaye PA, Akiana J, Camara P, Diop M, et al. Increase of malaria attacks among children presenting concomitant infection by Schistosoma mansoni in Senegal. Malar J 2004;3:43

12 Brun R, Schumacher R, Schmid C, Kunz C, Burri C. The phenomenon of treatment failures in human African trypanosomiasis. Trop Med Int Health 2001;6:906-14.

13 Sundar S, More DK, Singh MK, Singh VP, Sharma S, Makharia A, et al. Failure of pentavalent antimony in visceral leishmaniasis in India: report from the center of the Indian epidemic. Clin Infect Dis 2000;31:1104-7.

14 Osei-Atweneboana MY, Eng JK, Boakye DA, Gyapong JO, Prichard RK. Prevalence and intensity of Onchocerca volvulus infection and efficacy of ivermectin in endemic communities in Ghana: a two-phase epidemiological study. Lancet 2007;369:2021-9.
15 Trouiller P, Olliaro P, Torreele E, Orbinski J, Laing R, Ford N. Drug development for neglected diseases: a deficient market and a public-health policy failure. Lancet 2002;359:2188-94.

16 Moran M. A breakthrough in R\&D for neglected diseases: new ways to get the drugs we need. PLoS Med 2005;2:e302.

17 Salanti G, Kavvoura FK, loannidis JP. Exploring the geometry of treatment networks. Ann Intern Med 2008;148:544-53.

18 Salanti G, Higgins JPT, loannidis JPA. Evaluation of networks of randomized trials. Stat Meth Med Res 2008;17:279-301.

19 Mathers CD, Ezzati M, Lopez AD. Measuring the burden of neglected tropical diseases: the global burden of disease framework. PLoS Negl Trop Dis 2007;1:e114.

20 Torgerson PR, Keller K, Magnotta M, Ragland N. The global burden of alveolar echinococcosis. PLoS Negl Trop Dis 2010;4:e722.

21 Furst T, Keiser J, Utzinger J. Global burden of human food-borne trematodiasis: a systematic review and meta-analysis. Lancet Infect Dis 2012;12:210-21.

22 Knobel DL, Cleaveland S, Coleman PG, Fevre EM, Meltzer MI, Miranda ME, et al. Re-evaluating the burden of rabies in Africa and Asia. Bull World Health Organ 2005:83:360-8.

23 World Health Organization. WHO Metrics: disability-adjusted life year (DALY). Health statistics and health information systems. WHO, 2012.

24 World Health Organization. Buruli ulcer: provisional guidance on the role of specific antibiotics in the management of Mycobacterium ulcerans disease. WHO, 2012.

25 World Health Organization. Dengue - guidelines for diagnosis, treatment, prevention and control. WHO, 2009. 
26 Brunetti E, Kern P, Vuitton DA. Expert consensus for the diagnosis and treatment of cystic and alveolar echinococcosis in humans. Acta Trop 2010;114:1-16.

27 World Health Organization. Guide to eliminate leprosy as a public health problem. WHO, 2000.

28 Anonymous. Rabies. Guide for post-exposure prophylaxis. www.who.int/rabies/human/ postexp/en/.

29 World Health Organization. Trachoma control: a guide for programme managers. WHO, 2006.

30 Garcia HH, Evans CA, Nash TE, et al. Current consensus guidelines for treatment of neurocysticercosis. Clin Microbiol Rev 2002;15:747-56.

31 Anonymous. Drugs for parasitic infections. Treat Guide Med Lett 2007;5(suppl):e1-15.

32 Bethony J, Brooker S, Albonico M, Geiger SM, Loukas A, Diemert D, et al. Soil-transmitted helminth infections: ascariasis, trichuriasis, and hookworm. Lancet 2006:367:1521-32.

33 Norhayati M, Oothuman P, Azizi O, Fatmah MS. Efficacy of single dose albendazole on the prevalence and intensity of infection of soil-transmitted helminths in Orang Asli children in Malaysia. Southeast Asian J Trop Med Public Health 1997;28:563-9.

34 Vercruysse J, Behnke JM, Albonico M, Ame SM, Angebault C, Bethony JM, et al. Assessment of the anthelmintic efficacy of albendazole in school children in seven countries where soil-transmitted helminths are endemic. PLoS Negl Trop Dis 2011;5:e948.

35 Bartoloni A, Guglielmetti P, Cancrini G, Gamboa H, Roselli M, Nicoletti A, et al. Comparative efficacy of a single $400 \mathrm{mg}$ dose of albendazole or mebendazole in the treatment of nematode infections in children. Trop Geogr Med 1993;45:114-6.

36 Legesse M, Erko B, Medhin G. Comparative efficacy of albendazole and three brands of mebendazole in the treatment of ascariasis and trichuriasis. East Afr Med J 2004;81:134-8.

37 Keiser J, Utzinger J. Efficacy of current drugs against soil-transmitted helminth infections: systematic review and meta-analysis. JAMA 2008;299:1937-48.

38 Grencis RK, Cooper ES. Enterobius, trichuris, capillaria, and hookworm including ancylostoma caninum. Gastroenterol Clin North Am 1996;25:579-97.

39 Rossignol JF, Maisonneuve H. Benzimidazoles in the treatment of trichuriasis: a review. Ann Trop Med Parasitol 1984;78:135-44.

40 Hall A, Nahar Q. Albendazole and infections with Ascaris lumbricoides and Trichuris trichiura in children in Bangladesh. Trans R Soc Trop Med Hyg 1994:88:110-2.

41 Marti H, Haji HJ, Savioli L, Chwaya HM, Mgeni AF, Ameir JS, et al. A comparative trial of a single-dose ivermectin versus three days of albendazole for treatment of Strongyloides stercoralis and other soil-transmitted helminth infections in children. Am J Trop Med Hyg 1996;55:477-81.

42 Naquira C, Jimenez G, Guerra JG, Bernal R, Nalin DR, Neu D, et al. Ivermectin for human strongyloidiasis and other intestinal helminths. Am J Trop Med Hyg 1989;40:304-9.

43 Ottesen EA, Campbell WC. Ivermectin in human medicine. J Antimicrob Chemother 1994;34:195-203

44 Flohr C, Tuyen LN, Lewis S, Minh TT, Campbell J, Britton J, et al. Low efficacy of mebendazole against hookworm in Vietnam: two randomized controlled trials. Am J Trop Med Hyg 2007;76:732-6.

45 Albonico M, Bickle Q, Ramsan M, Montresor A, Savioli L, Taylor M. Efficacy of mebendazole and levamisole alone or in combination against intestinal nematode infections after repeated targeted mebendazole treatment in Zanzibar. Bull World Health Organ 2003:81:343-52

46 Sacko M, De Clercq D, Behnke JM, Gilbert FS, Dorny P, Vercruysse J. Comparison of the efficacy of mebendazole, albendazole and pyrantel in treatment of human hookworm infections in the southern region of Mali, West Africa. Trans $R$ Soc Trop Med Hyg 1999;93:195-203.

47 Igual-Adell R, Oltra-Alcaraz C, Soler-Company E, Sanchez-Sanchez P, Matogo-Oyana $J$, Rodriguez-Calabuig D. Efficacy and safety of ivermectin and thiabendazole in the treatment of strongyloidiasis. Expert Opin Pharmacother 2004;5:2615-9.

48 Archibald LK, Beeching NJ, Gill GV, Bailey JW, Bell DR. Albendazole is effective treatment for chronic strongyloidiasis. Q J Med 1993;86:191-5.

49 Hoerauf A. Filariasis: new drugs and new opportunities for lymphatic filariasis and onchocerciasis. Curr Opin Infect Dis 2008;21:673-81.

50 Ross AG, Bartley PB, Sleigh AC, Olds GR, Li Y, Williams GM, et al. Schistosomiasis. N Engl J Med 2002;346:1212-20.

51 Tisch DJ, Michael E, Kazura JW. Mass chemotherapy options to control lymphatic filariasis: a systematic review. Lancet Infect Dis 2005;5:514-23.

52 Alvar J, Croft S, Olliaro P. Chemotherapy in the treatment and control of leishmaniasis. Adv Parasitol 2006;61:223-74.

53 Bern C, Adler-Moore J, Berenguer J, Boelaert M, den Boer M, Davidson RN, et al. Liposomal amphotericin B for the treatment of visceral leishmaniasis. Clin Infect Dis 2006;43:917-24

54 Sundar S, Mehta H, Suresh AV, Singh SP, Rai M, Murray HW. Amphotericin B treatment for Indian visceral leishmaniasis: conventional versus lipid formulations. Clin Infect Dis 2004:38:377-83.

55 Den Boer M, Davidson RN. Treatment options for visceral leishmaniasis. Expert Rev Anti Infect Ther 2006;4:187-97.

56 Sundar S, Jha TK, Thakur CP, Engel J, Sindermann H, Fischer C, et al. Oral miltefosine for Indian visceral leishmaniasis. N Engl J Med 2002;347:1739-46.

57 Herwaldt BL, Berman JD. Recommendations for treating leishmaniasis with sodium stibogluconate (Pentostam) and review of pertinent clinical studies. Am J Trop Med Hyg 1992:46:296-306.

58 Evans TG. Leishmaniasis. Infect Dis Clin North Am 1993;7:527-46.

59 Soto J, Arana BA, Toledo J, Rizzo N, Vega JC, Diaz A, et al. Miltefosine for new world cutaneous leishmaniasis. Clin Infect Dis 2004;38:1266-72.

60 Reithinger R, Dujardin JC, Louzir H, Pirmez C, Alexander B, Brooker S. Cutaneous leishmaniasis. Lancet Infect Dis 2007;7:581-96.

61 Davidson RN. Practical guide for the treatment of leishmaniasis. Drugs 1998;56:1009-18.

62 Sosa Estani S, Segura EL, Ruiz AM, Velazquez E, Porcel BM, Yampotis C. Efficacy of chemotherapy with benznidazole in children in the indeterminate phase of Chagas' disease. Am J Trop Med Hyg 1998;59:526-9.

63 De Andrade AL, Zicker F, de Oliveira RM, Almeida Silva S, Luquetti A, Travassos LR, et al. Randomised trial of efficacy of benznidazole in treatment of early Trypanosoma cruzi infection. Lancet 1996;348:1407-13
64 Bern C, Montgomery SP, Herwaldt BL, Rassi A Jr, Marin-Neto JA, Dantas RO, et al. Evaluation and treatment of chagas disease in the United States: a systematic review. JAMA 2007:298:2171-81.

65 Harinasuta T, Pungpak S, Keystone JS. Trematode infections. Opisthorchiasis, clonorchiasis, fascioliasis, and paragonimiasis. Infect Dis Clin North Am 1993;7:699-716.

66 Jong EC, Wasserheit JN, Johnson RJ, Carberry WL, Agosti J, Dunning S, et al. Praziquantel for the treatment of clonorchis/opisthorchis infections: report of a double-blind, placebo-controlled trial. J Infect Dis 1985;152:637-40.

67 Pungpak S, Radomyos P, Radomyos BE, Schelp FP, Jongsuksuntigul P, Bunnag D. Treatment of Opisthorchis viverrini and intestinal fluke infections with praziquantel. Southeast Asian J Trop Med Public Health 1998;29:246-9.

68 Arjona R, Riancho JA, Aguado JM, Salesa R, Gonzalez-Macias J. Fascioliasis in developed countries: a review of classic and aberrant forms of the disease. Medicine (Baltimore) 1995;74:13-23.

69 Bacq Y, Besnier JM, Duong TH, Pavie G, Metman EH, Choutet P. Successful treatment of acute fascioliasis with bithionol. Hepatology 1991;14:1066-9.

70 Farag HF, Salem A, el-Hifni SA, Kandil M. Bithionol (Bitin) treatment in established fascioliasis in Egyptians. J Trop Med Hyg 1988;91:240-4.

71 Bunnag D, Radomyos P, Harinasuta T. Field trial on the treatment of fasciolopsiasis with praziquantel. Southeast Asian J Trop Med Public Health 1983;14:216-9.

72 Keiser J, Engels D, Buscher G, Utzinger J. Triclabendazole for the treatment of fascioliasis and paragonimiasis. Expert Opin Investig Drugs 2005;14:1513-26.

73 Ripert C, Couprie B, Moyou R, Gaillard F, Appriou M, Tribouley-Duret J. Therapeutic effect of triclabendazole in patients with paragonimiasis in Cameroon: a pilot study. Trans R Soc Trop Med Hyg 1992;86:417.

74 Calvopina M, Guderian RH, Paredes W, Chico M, Cooper PJ. Treatment of human pulmonary paragonimiasis with triclabendazole: clinical tolerance and drug efficacy. Trans R Soc Trop Med Hyg 1998;92:566-9.

75 Priotto G, Kasparian S, Mutombo W, Ngouama D, Ghorashian S, Arnold U, et al. Nifurtimox-eflornithine combination therapy for second-stage African Trypanosoma bruce gambiense trypanosomiasis: a multicentre, randomised, phase III, non-inferiority trial. Lancet 2009;374:56-64.

76 Priotto G, Kasparian S, Ngouama D, Ghorashian S, Arnold U, Ghabri S, et al. Nifurtimox-eflornithine combination therapy for second-stage Trypanosoma brucei gambiense sleeping sickness: a randomized clinical trial in Congo. Clin Infect Dis 2007; $45: 1435-42$

77 Schmid C, Richer M, Bilenge CM, Josenando T, Chappuis F, Manthelot CR, et al. Effectiveness of a 10-day melarsoprol schedule for the treatment of late-stage human African trypanosomiasis: confirmation from a multinational study (IMPAMEL II). $J$ Infect Dis 2005:191:1922-31.

78 Duke BO. Onchocerciasis. Br Med Bull 1972;28:66-71.

79 Aziz MA. Chemotherapeutic approach to control of onchocerciasis. Rev Infect Dis 1986;8:500-4

80 Most $\mathrm{H}$. Treatment of common parasitic infections of man encountered in the United States. I. N Engl J Med 1972;287:495-8.

81 World Health Organization. Chemotherapy of leprosy for control programmes. World Health Organ Tech Rep Ser 1982;675:1-33.

82 Zhang L. Leprosy in China. Nihon Hansenbyo Gakkai Zasshi 2003;72:209-15.

83 Lwin K, Myint T, Gyi MM, Thein M, Shwe T, Sein KN. Leprosy control in Myanmar 1952-2003-a success story. Lepr Rev 2005;76:77-86.

84 Ottesen EA, Ismail MM, Horton J. The role of albendazole in programmes to eliminate lymphatic filariasis. Parasitol Today 1999;15:382-6.

85 Cao WC, Van der Ploeg CP, Plaisier AP, van der Sluijs IJ, Habbema JD. Ivermectin for the chemotherapy of bancroftian filariasis: a meta-analysis of the effect of single treatment. Trop Med Int Health 1997;2:393-403.

86 Ribeiro-dos-Santos G, Verjovski-Almeida S, Leite LCC. Schistosomiasis-a century searching for chemotherapeutic drugs. Parasitology Res 2006:99:505-21.

87 Estellat C, Ravaud P. Lack of head-to-head trials and fair control arms: randomized controlled trials of biologic treatment for rheumatoid arthritis. Arch Intern Med 2012;172:237-44

88 Thase ME. Do antidepressants really work? A clinicians' guide to evaluating the evidence. Curr Psychiatry Rep 2008;10:487-94.

89 Heres S, Davis J, Maino K, Jetzinger E, Kissling W, Leucht S. Why olanzapine beats risperidone, risperidone beats quetiapine, and quetiapine beats olanzapine: an exploratory analysis of head-to-head comparison studies of second-generation antipsychotics. Am J Psychiatry 2006;163:185-94.

90 Bero L, Oostvogel F, Bacchetti P, Lee K. Factors associated with findings of published trials of drug-drug comparisons: why some statins appear more efficacious than others. PLoS Med 2007:4:e184.

91 Lathyris DN, Patsopoulos NA, Salanti G, loannidis JP. Industry sponsorship and selection of comparators in randomized clinical trials. Eur J Clin Invest 2010;40:172-82.

92 Bill and Melinda Gates Foundation. Leading global health organizations receive $\$ 46.7$ Million from Gates foundation to integrate programs fighting neglected tropical diseases. Grantee Announcement, 2006.

93 Finkelstein JL, Schleinitz MD, Carabin H, McGarvey ST. Decision-model estimation of the age-specific disability weight for schistosomiasis japonica: a systematic review of the literature. PLoS Negl Trop Dis 2008;2:e158.

94 Centers for Disease Control. Progress toward global eradication of dracunculiasis, January 2010-June 2011. MMWR Morb Mortal Wkly Rep 2011;60:1450-3.

Accepted: 18 September 2012

\section{Cite this as: BMJ 2012;345:e6512}

This is an open-access article distributed under the terms of the Creative Commons Attribution Non-commercial License, which permits use, distribution, and reproduction in any medium, provided the original work is properly cited, the use is non commercial and is otherwise in compliance with the license. See: http://creativecommons.org/licenses/by$\mathrm{nc} / 2.0 /$ and http://creativecommons.org/licenses/by-nc/2.0/legalcode. 


\section{Tables}

\section{Table 1| Characteristics of included randomised controlled trials}

\begin{tabular}{|c|c|}
\hline Characteristic & No (\%) \\
\hline Examines primary disease & $854(88.0)$ \\
\hline Examines complication of disease & $117(12.0)$ \\
\hline \multicolumn{2}{|c|}{ Location of study sites (WHO Regions) ${ }^{*}$ : } \\
\hline Africa & $283(27.6)$ \\
\hline Southeast Asia & $276(26.9)$ \\
\hline Americas & $201(19.6)$ \\
\hline Eastern Mediterranean & $132(12.9)$ \\
\hline Western Pacific & $96(9.4)$ \\
\hline Europe & $38(3.7)$ \\
\hline \multicolumn{2}{|l|}{ No of sites: } \\
\hline Single centre & $945(97.3)$ \\
\hline Multicentre & $26(2.7)$ \\
\hline Cluster randomised trials & $27(2.8)$ \\
\hline Crossover trials & $15(1.5)$ \\
\hline \multicolumn{2}{|l|}{ Funding: } \\
\hline Contains explicit statement & $565(58.2)$ \\
\hline Any industry funding & $107(11.0)$ \\
\hline Any public funding & $388(40.0)$ \\
\hline Any charity or foundation funding & $184(18.9)$ \\
\hline Any university or hospital funding & $79(8.1)$ \\
\hline No explicit statement of funding & $404(41.6)$ \\
\hline Unable to ascertain & $2(<1)$ \\
\hline \multicolumn{2}{|c|}{ Trial size (total No randomised participants)†: } \\
\hline Mean & 191.0 \\
\hline Median (IQR) & $89(42-184)$ \\
\hline \multicolumn{2}{|l|}{ Follow-up (years): } \\
\hline$\leq 1$ & $772(79.5)$ \\
\hline$>1$ & $199(20.5)$ \\
\hline
\end{tabular}

IQR=interquartile range.

*Total number of study sites is more than 971 because of multicentre trials.

†Calculated with "units of randomisation" in each trial, including number of clusters in cluster randomised trials and number of randomised units in trials in which unit of randomisation was not individual (for example, skin lesions in leishmaniasis or eyes in trachoma). It slightly underestimates number of individual participants. 
Table 2| Number of randomised controlled trials published before and after 2000 for each disease

Buruli ulcer

1

\begin{tabular}{lcc}
\hline Chagas disease & 15 & 7 \\
\hline Cysticercosis & 12 & 20 \\
\hline Dengue & 9 & 15 \\
\hline Dracunculiasis & 9 & 0 \\
\hline Echinococcosis & 6 & 9 \\
\hline Foodborne trematode infections & 9 & 5 \\
\hline Geohelminth infections & 113 & 47 \\
\hline Human African trypanosomiasis & 1 & 9 \\
\hline Leishmaniasis & 82 & 102 \\
\hline Leprosy & 93 & 27 \\
\hline Lymphatic filariasis & 40 & 33 \\
\hline Onchocerciasis & 52 & 10 \\
\hline Rabies & 24 & 21 \\
\hline Schistosomiasis & 109 & 33 \\
\hline Trachoma & 34 & 20
\end{tabular}




\begin{tabular}{|c|c|c|c|c|}
\hline & No of trials* & $\begin{array}{l}\text { No exclusively examining } \\
\text { primary disease }\end{array}$ & Total sample size $†$ & $\begin{array}{l}\text { Annual global burden of } \\
\text { disease in } 1000 \text { s of DALYs }\end{array}$ \\
\hline Leishmaniasis & 184 & 179 & 23039 & 2090 \\
\hline Geohelminth infections & 160 & 133 & 46887 & 3796 \\
\hline Ascariasis & - & $79 \ddagger$ & - & - \\
\hline Hookworm & - & $77 \ddagger$ & - & - \\
\hline Trichuriasis & - & $66 \ddagger$ & - & - \\
\hline Strongyloidiasis & - & $14 \ddagger$ & - & - \\
\hline Schistosomiasis & 142 & 114 & 35026 & 1702 \\
\hline Leprosy & 120 & 77 & 14772 & 198 \\
\hline Lymphatic filariasis & 73 & 73 & 9996 & 5777 \\
\hline Onchocerciasis & 62 & 53 & 25182 & 484 \\
\hline Trachoma & 54 & 29 & 10420 & 2329 \\
\hline Rabies§ & 45 & 13 & 6756 & 1780 \\
\hline Cysticercosis & 32 & 32 & 2872 & - \\
\hline Dengue & 24 & 4 & 2374 & 616 \\
\hline American trypanosomiasis & 22 & 8 & 1772 & 667 \\
\hline Echinococcosis & 15 & 15 & 1064 & 666 \\
\hline Foodborne trematode infections & 14 & 14 & 1306 & 665 \\
\hline Fascioliasis & & $5 \ddagger$ & - & - \\
\hline Opisthorchiasis & & $4 \ddagger$ & 一 & 一 \\
\hline Clonorchiasis & & $3 \ddagger$ & - & - \\
\hline Paragonimiasis & & $2 \ddagger$ & - & - \\
\hline Fasciolopsiasis & & $1 \neq$ & - & - \\
\hline Human African trypanosomiasis $\mathbb{T}$ & 10 & 10 & 2840 & 1525 \\
\hline Dracunculiasis & 9 & 9 & 798 & - \\
\hline Buruli ulcer & 5 & 5 & 337 & - \\
\hline
\end{tabular}

*Includes both primary disease and its complications.

†Calculated with "units of randomisation" in each trial, including number of clusters in cluster randomised trials and number of randomised units in trials in which unit of randomisation was not individual (for example, skin lesions in leishmaniasis or eyes in trachoma). It slightly underestimates number of individual participants. ¥Sum of individual categories is greater than total number of trials because of trials that examined more than disease.

§Thirteen trials examined post-exposure prophylaxis after appropriate exposure, 32 others were simulated prophylaxis trials

ףEarly and late stage Trypanosoma brucei gambiense. 
Table 4| Number of trials examining primary disease, number of regimens tested, and amount of evidence from randomised clinical trials supporting first and second line treatments in 16 neglected tropical diseases

\begin{tabular}{|c|c|c|c|}
\hline & Trials & Regimens & No of trials/sample size supporting first and second line treatments \\
\hline Schistosomiasis & 114 & 34 & Praziquantel (76/14488); oxamniquine (23/2419) \\
\hline Ascariasis & 79 & 37 & $\begin{array}{l}\text { mebendazole (24/5865); albendazole (33/7526); pyrantel pamoate (16/1838); } \\
\text { levamisole (12/1254); ivermectin (5/1432); piperazine citrate (5/223); nitazoxanide } \\
(1 / 35)\end{array}$ \\
\hline Hookworm & 77 & 29 & $\begin{array}{l}\text { Albendazole (40/7106); mebendazole (27/5790); pyrantel pamoate (16/1429); } \\
\text { levamisole }(3 / 400)\end{array}$ \\
\hline Trichuriasis & 66 & 24 & Mebendazole (25/6321); albendazole (41/8739); ivermectin (5/1432) \\
\hline Strongyloidiasis & 14 & 6 & Ivermectin (6/803); albendazole (11/1066) \\
\hline Lymphatic filariasis & 73 & 17 & Diethylcarbamazine plus albendazole (15/1953); diethylcarbamazine (44;/2901) \\
\hline Visceral leishmaniasis & 67 & 29 & $\begin{array}{l}\text { Pentavalent antimonials (28/3026); liposomal amphotericin (7/758); miltefosine } \\
\text { (5/754); amphotericin B deoxycholate (23/3999); paromomycin (5/1098); } \\
\text { amphotericin B colloidal dispersion (1/405); amphotericin B lipid complex (5/284) }\end{array}$ \\
\hline Old World cutaneous leishmaniasis & 61 & 35 & Pentavalent antimonials (16/863); heat treatment (3/224) \\
\hline New World cutaneous leishmaniasis & 44 & 22 & Pentavalent antimonials (39/1589); miltefosine (5/398); pentamidine (2/55) \\
\hline Mucocutaneous leishmaniasis & 7 & 7 & Pentavalent antimonials (5/157); amphotericin B deoxycholate (1/10) \\
\hline Onchocerciasis & 53 & 26 & Ivermectin (27/5398); doxycycline plus ivermectin (2/150) \\
\hline Multibacillary leprosy & 56 & 55 & Rifampin, dapsone plus clofazimine (16/846) \\
\hline Paucibacillary leprosy & 21 & 16 & Rifampin plus dapsone (14/2299) \\
\hline Trachoma & 29 & 22 & Oral azithromycin (8/871); ophthalmic tetracyclines (14/1849) \\
\hline Rabies & 13 & 17 & $\begin{array}{l}\text { PVCV IM (3/399); PCECV ID (4/171); PCECV IM (4/134); CPVCV IM (1/200); } \\
\text { PDEV IM (1/52); HDCV IM (1/10); CPVCV IM plus rabies Ig ( } 2 / 255) ; \text { PCECV IM } \\
\text { plus rabies Ig (2/159); PVCV ID (2/374); HDCV ID rabies Ig (1/78); HDCV IM } \\
\text { plus rabies Ig (1/39); PVCV IM plus rabies Ig (1/47) }\end{array}$ \\
\hline Cysticercosis & 32 & 7 & Albendazole plus corticosteroids (12/690); praziquantel plus corticosteroids (2/64) \\
\hline American trypanosomiasis & 8 & 5 & Benznidazole (5/429); nifurtimox (1/27) \\
\hline Echinococcosis & 15 & 8 & $\begin{array}{l}\text { Surgery alone ( } 8 / 279) \text {; surgery plus albendazole }(5 / 209) \text {; albendazole }(4 / 224) \text {; } \\
\text { percutaneous drainage plus albendazole }(2 / 37) \text {; placebo or no treatment }(2 / 64) \\
\text { mebendazole (3/204) }\end{array}$ \\
\hline Human African trypanosomiasis* & 10 & 7 & $\begin{array}{l}\text { Melarsoprol (5/1865); nifurtimox plus eflornithine (3/211); eflornithine ( } 4 / 536) \text {; } \\
\text { pentamidine }(1 / 52)\end{array}$ \\
\hline Dracunculiasis & 9 & 6 & No recommended first line treatment against disease among tested agents \\
\hline Buruli ulcer & 5 & 8 & $\begin{array}{l}\text { Streptomycin plus rifampin (1/76); streptomycin, rifampin plus clarithromycin } \\
\text { (1/75); streptomycin plus rifampin followed by excision (1/16) }\end{array}$ \\
\hline Dengue or dengue hemorrhagic fever & 4 & 6 & No recommended first line treatment against disease among tested agents \\
\hline \multicolumn{4}{|l|}{ Foodborne trematode infections: } \\
\hline Fascioliasis & 5 & 4 & Triclabendazole (4/399); nitazoxanide (1/80) \\
\hline Opisthorchiasis & 4 & 7 & Praziquantel $(3 / 140)$ \\
\hline Clonorchiasis & 3 & 5 & Praziquantel (3/63) \\
\hline Paragonimiasis & 2 & 2 & Praziquantel (1/15); triclabendazole (2/201) \\
\hline Fasciolopsiasis & 1 & 1 & Praziquantel $(1 / 85)$ \\
\hline
\end{tabular}

$\mathrm{ID}=$ intradermal; IM=intramuscular; $\mathrm{PVCV}=$ purified Vero cell vaccine; $\mathrm{PCECV}=$ purified chick embryo cell vaccine; $\mathrm{CPVCV}=$ chromatographically purified Vero cell vaccine; $\mathrm{HDCV}$ =human diploid cell rabies vaccine; $P D E V=$ purified duck embryo vaccine; Ig=immunoglobulin.

*Early and late stage Trypanosoma brucei gambiense. 
Table 5| Network metrics for 10 disease networks shown in figure 3

C score $\mathrm{P}$ value Diversity (PIE) score

\begin{tabular}{lcc} 
New World cutaneous leishmaniasis & 0.13 & 0.82 \\
\hline Old World cutaneous leishmaniasis & 0.031 & 0.92 \\
\hline Visceral leishmaniasis & 0.039 & 0.88 \\
\hline Ascariasis & $<0.001$ & 0.90 \\
\hline Trichuriasis & 0.003 & 0.86 \\
\hline Hookworm & 0.013 & 0.94 \\
\hline Schistosomiasis & 0.38 & 0.83 \\
\hline Multibacillary leprosy & $<0.001$ & 0.94 \\
\hline Lymphatic filariasis & 0.014 & 0.82 \\
\hline Onchocerciasis & 0.07 & 0.82
\end{tabular}




\section{Figures}

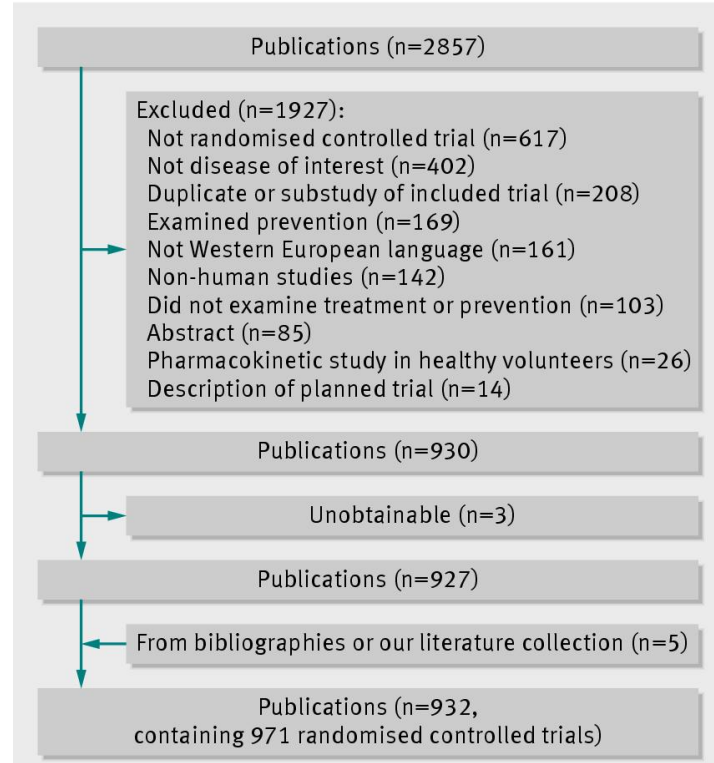

Fig 1 Schematic of literature search
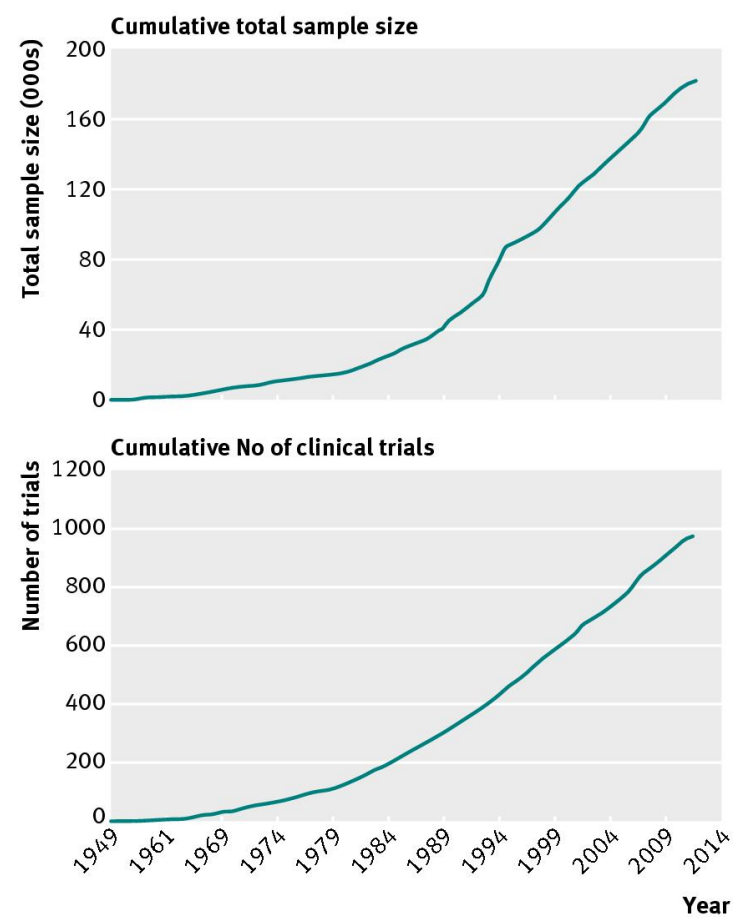

Fig 2 Total sample size and number of clinical trials over time 

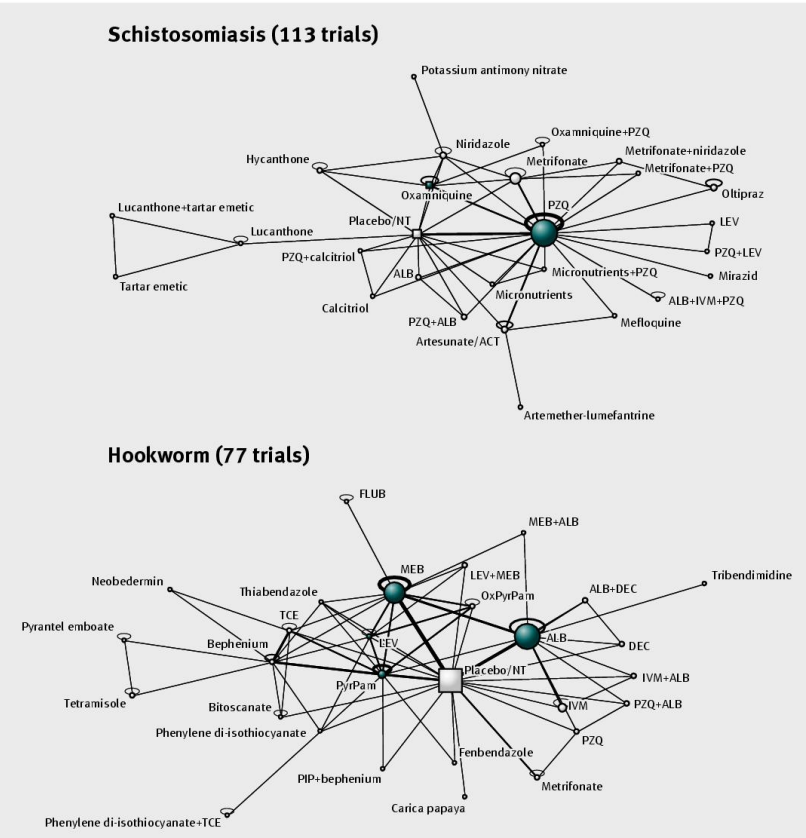

Lymphatic filariasis (72 trials)
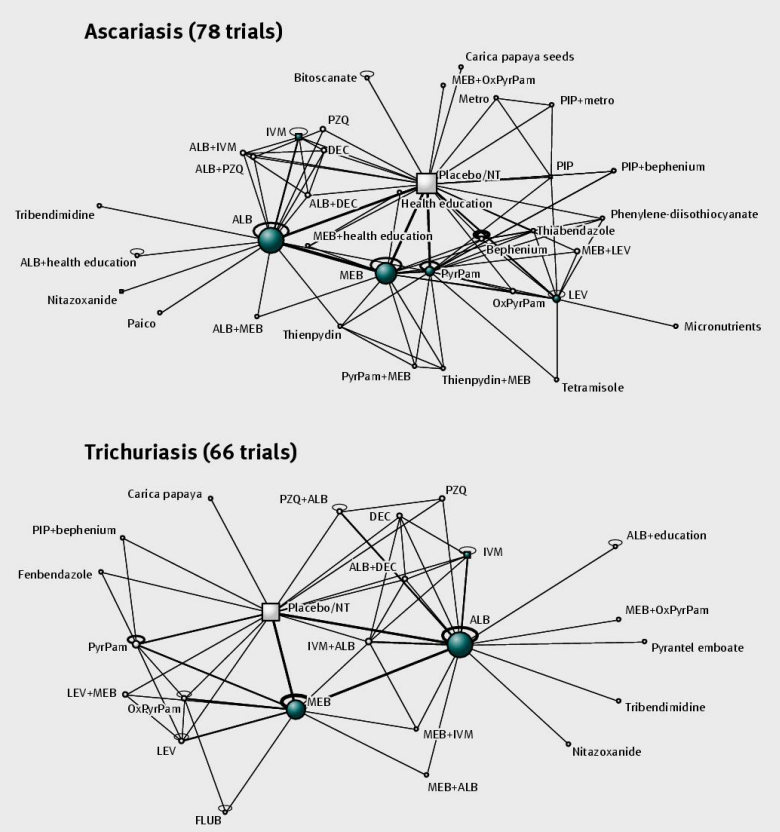

Visceral leishmaniasis (64 trials)

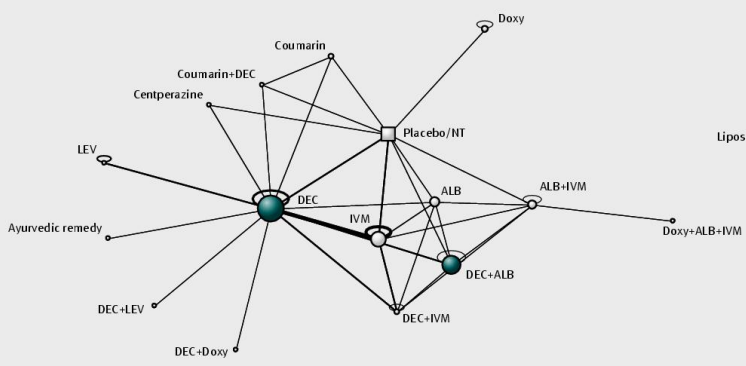

old World cutaneous leishmaniasis (60 trials)

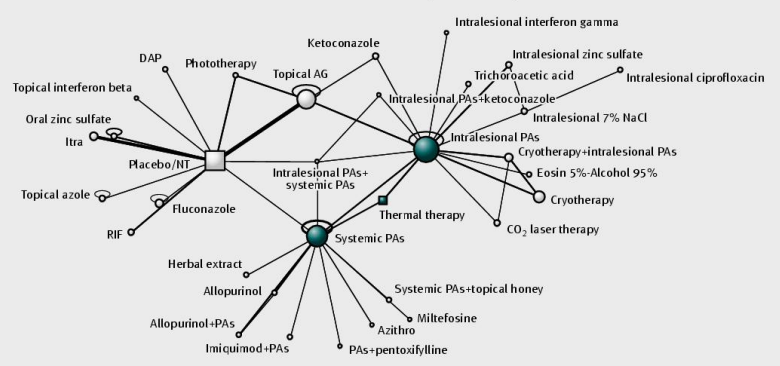

Onchocerciasis ( 53 trials)

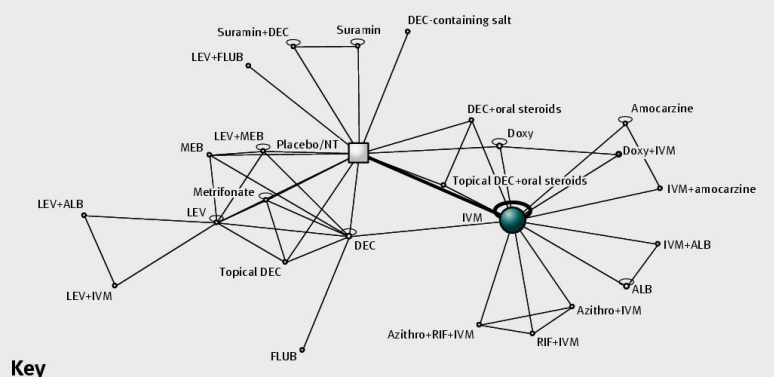

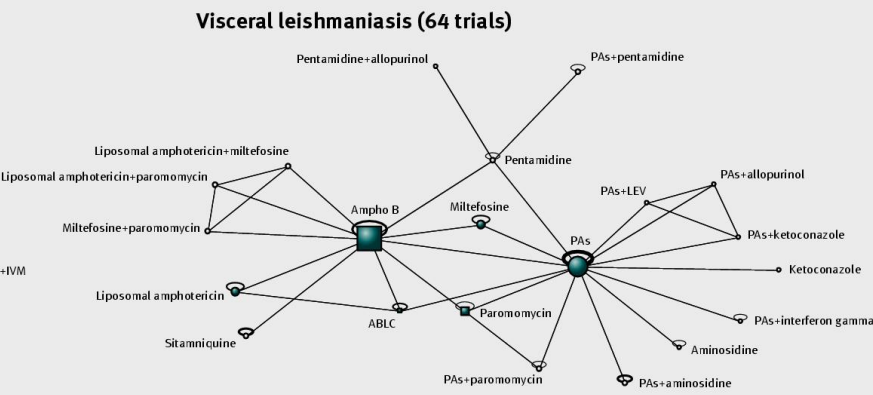

New World cutaneous leishmaniasis (44 trials)

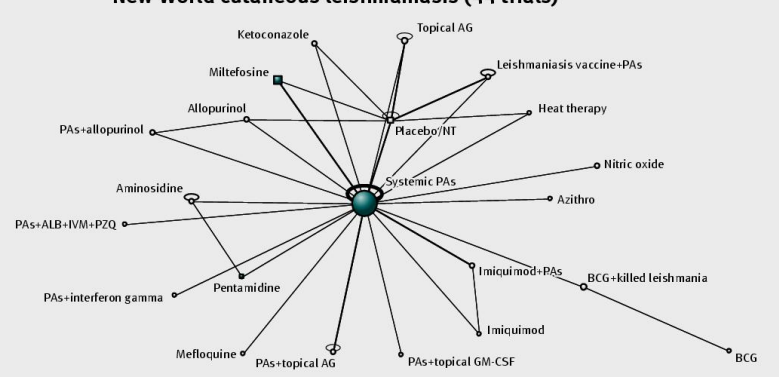

Multibacillary leprosy (52 trials)

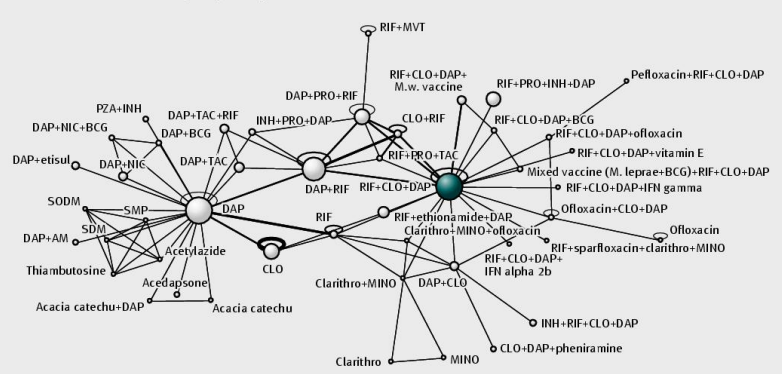

- First line treatments $\square$ Second line treatments $O$ All other treatments $\square$ Placebo or no treatment

Fig 3 Network diagrams for diseases with more than 40 randomised controlled trials. Node size is proportional to total sample size and line thickness is proportional to number of comparisons. Scale of nodes is different between different networks; see appendix table 3 for exact information on number of participants on each regimen. $A B L C=a m p h o t e r i c i n ~ B$ lipid complex; ACT=artesunate combination treatment (includes artesunate+sulfalene, artesunate+mefloquine, artesunate+sulfadoxine-pyrimethamine, artesunate+sulfamethoxypyrazine-pyrimethamine, artesunate-amodiaquine and artesunate+praziquantel); $\mathrm{ALB}=$ albendazole; $\mathrm{AM}=$ antigen marianum; Azithro=azithromycin; Clarithro=clarithromycin; $\mathrm{CLO}=$ clofazimine; $\mathrm{DAP}=$ dapsone; $\mathrm{DEC}=$ diethylcarbamazine; Doxy=doxycycline; FLUB=flubendazole;

GM-CSF=granulocyte-macrophage colony-stimulating factor; IFN=interferon; INH=isoniazid; Itra=itraconazole; IVM=ivermectin; $\mathrm{LEV}=$ =levamisole; $\mathrm{MEB}=$ mebendazole; Metro=metronidazole; $\mathrm{MINO}=$ minocycline; $\mathrm{MVT}=$ multivitamin; NIC=nicotinamide; 
$\mathrm{NT}=$ no treatment; OxPyrPam=oxantel-pyrantel pamoate; $\mathrm{PAs}=$ pentavalent antimonials; $\mathrm{PIP}=$ piperazine; $\mathrm{PRO}=$ prothionamide; PyrPam=pyrantel pamoate; $P Z A=$ pyrazinamide; $P Z Q=$ praziquantel; RIF=rifampin; RIF+CLO+DAP=rifampin, clofazimine, and dapsone; $\mathrm{SDM}=$ sulfadimethoxine; $\mathrm{SMP}=$ sulfamethoxypyridazine; $\mathrm{SODM}=$ sulforthodimethoxine; TCE=tetrachloroethylene; TAC=thiacetazone; Topical $A G=$ topical aminoglycoside 